\title{
ACUTE LeUKemia Classification Using Convolution NeUral NeTWORK IN Clinical DECISION SUPPORT SYSTEM
}

\author{
Thanh.TTP ${ }^{1}$, Giao N. Pham ${ }^{1}$, Jin-Hyeok Park ${ }^{1}$, Kwang-Seok Moon ${ }^{2}$, Suk-Hwan \\ Lee $^{3}$, and Ki-Ryong Kwon ${ }^{1}$ \\ ${ }^{1}$ Dept. of IT Convergence and Applications Eng., \\ ${ }^{2}$ Dept. Electronics Eng.,Pukyong National University, Busan, Korea \\ ${ }^{3}$ Dept. of Information Security, Tongmyong University, Busan, Korea
}

\begin{abstract}
Leukemia induced death has been listed in the top ten most dangerous mortality basis for human being. Some of the reason is due to slow decision-making process which caused suitable medical treatment cannot be applied on time. Therefore, good clinical decision support for acute leukemia type classification has become a necessity. In this paper, the author proposed a novel approach to perform acute leukemia type classification using convolution neural network $(C N N)$ classifier. Our experimental result only covers the first classification process which shows an excellent performance in differentiating normal and abnormal cells. Further development is needed to prove the effectiveness of second neural network classifier.
\end{abstract}

\section{KEYWORDS}

Acute Leukemia Classification, Convolution neural network, Clinical decision support system

\section{INTRODUCTION}

Leukemia is a group of cancers that begin in the bone marrow and result in high numbers of abnormal white blood cells. It attacks white blood cells which responsible for immune system and disease prevention in the human body. There are four main types of Leukemia which are classified based on severity level and infected cells type - acute lymphoblastic leukemia (ALL), acute myeloid leukemia (AML), chronic lymphocytic leukemia (CLL) and chronic myeloid leukemia (CML). In this paper, we would like to propose a novel approach to perform acute leukemia classification based on Convolution Neural Network (CNN). This method provides an excellent performance in classification process that reaches $96.43 \%$ of accuracy to discriminate normal and abnormal cell images from given database.

\section{RELATED WORK}

\subsection{OVERVIEW OF LEUKEMIA}

\section{A. Acute Lymphocytic Leukemia (ALL)}

Acute Lymphocytic Leukemia (ALL) is one between two classes of acute leukemia which develop from early (immature) forms of lymphocytes cells in bone marrow. ALL is recorded as the most common of the 4 major types among children but the least common types in adults, but most deaths occur in adults which are understandable because children's bodies can often handle aggressive treatment better than adult's. Most leukemia infected white blood cells has average Natarajan Meghanathan et al. (Eds) : ICAITA, Signal, SAI - 2017 
size about two times the red blood cells that surround them, while normal white blood cells have an average size that similar with the surrounding red blood cells. In most of ALL infected cells, the nucleus area occupies almost $80-90 \%$ of the whole cell and left about $20-30 \%$ of cell area for the cytoplasm. The surface of ALL cell is smooth.

\section{B. Acute Myeloid Leukemia (AML)}

Acute Myeloid Leukemia is developed from early (immature) forms of myeloid cells in bone marrow. AML cases generally occur in older people and uncommon before the age of 45. AML cases hold the most number of deaths among the 4 types of leukemia. The nucleus area is about $50-60 \%$ of the whole cell area and about $40-50 \%$ cell area is cytoplasm. Sometimes short purple stripes which called "Auer rods" can be found in the cytoplasm area and it makes AML identification become easier.

\section{Chronic Lymphocytic Leukemia (CLL)}

Chronic Lymphocytic Leukemia is a type of leukemia that attacks the lymphocytes, different from ALL which attack the immature form of lymphocytes. CLL cell has a similar characteristic with ALL with a small area of cytoplasm and almost $80-90 \%$ of the cell area is occupied by nucleus area. The difference between ALL and CLL is the existence of nucleoli inside the nucleus area. Because CLL is basically mature cells which development has been completed, there should not be any nucleolus detected inside CLL cells.

\section{Chronic Myeloid Leukemia (CML)}

Chronic Myeloid Leukemia is another type of chronic leukemia that attacks the myeloid cells and has a similar behavior like CLL, especially in the development state. The average age at diagnosis of CML is around 64 years old. CML cell has a similar characteristic with AML in terms of cytoplasm area ratio, CML also show a big portion of cytoplasm in the cell and most of CML cells are fully developed into mature white blood cells which make it as the most differentiable cells among the four leukemia types. CML nucleus has developed into differentiable shape and texture unlike AML which in most cases the shape is round and under developed.

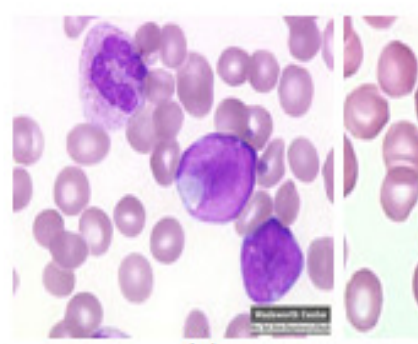

(a)

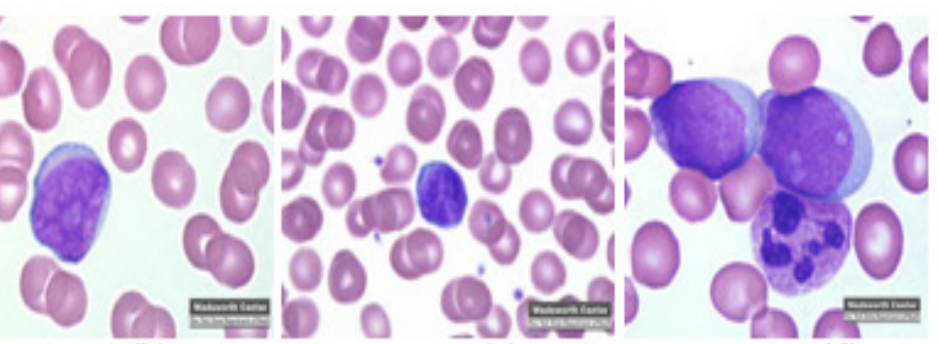

(b) (c) (d)

Figure 1. (a) Sample images of Acute Lymphocytic Leukemia, (b) Acute Myeloid Leukemia, (c) Chronic Lymphocytic Leukemia, and (d) Chronic Myeloid Leukemia.

\subsection{ReCent Leukemia Detection And Classification Methods}

The researches about leukemia classification in recent years based on computer vision. The most common algorithm in this approach consists of several rigid steps: image pre-processing, clustering, morphological filtering, segmentation, feature selection or extraction, classification, and evaluation [1] [2]. Almost authors have used machine learning techniques to detect blood cell 
in images and to classify cells in images. They extract features representing points, regions, or objects of interest and then use those features to train a model to classify or learn patterns in the image data. Feature extraction usually involves processing each image with some imageprocessing operations, such as calculating gradient to extract the discriminative information from each image. In this paper, we used the method of deep learning to learn characteristics of leukemia shape to classify normal and abnormal cell images.

\section{Proposed Method}

In this paper, we will use a Convolution Neural Network (CNN) to perform classification and extract features from raw images.

\subsection{ARCHITECTURE OF CNN}

In this work, we proposed a network contains 4 layers. The first 3 layers for detecting features and the other two layers (Fully connected and Softmax) are for classifying the features. The input image has the size [50x50x3]. The receptive field (or the filter size) is 5x5. The stride is 1 then we move the filters one pixel at a time. The zero-padding is 2 . It will allow us to control the spatial size of the output image (we will use it to exactly preserve the spatial size of the input volume so the input and output width and height are the same). During the experiment, we found that in our case, altering the size of original image during the convolution lead to decrease the accuracy about $40 \%$. Thus the output image after convolution layer 1 has the same size with the input image.

The convolution layer 2 has the same structure with the convolution layer 1 . The filter size is $5 \times 5$, the stride is 1 and the zero-padding is 2 . The number of feature maps (the channel or the depth) in our case is 30. If the number of feature maps is lower or higher than 30, the accuracy will decrease $50 \%$. By experiment, we found the accuracy also decrease $50 \%$ if we remove Convolution layer 2.

The Max-Pooling layer $25 \times 25$ has Filter size is 2 and stride is 2 . The fully connected layer has 2 neural. Finally, we use the Softmax layer for the classification.

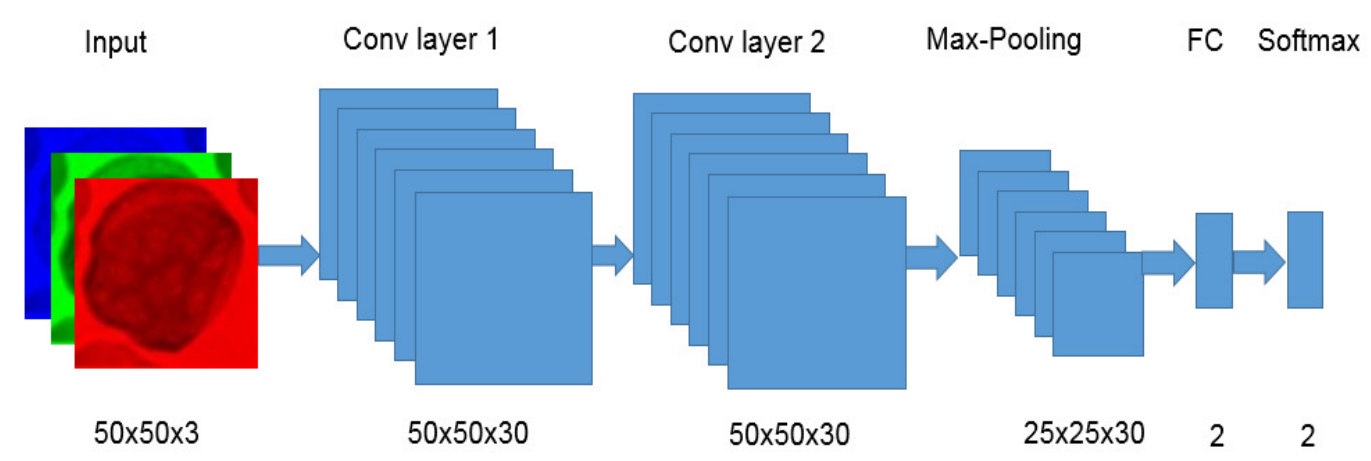

Figure 2. The architecture of network.

\section{EXPERIMENT AND RESULT}

In this experiment, the original ALL-IDB1 image database which consists of 108 cell image [3] (59 normal cell images and 49 abnormal cell images) was used. The number of labelled training data and unlabelled testing data are shown in Table 1. 
The experiment conducts on Matlab and the accuracy rate of the recognition of leukemia by our proposed CNN model achieved $96.43 \%$.

Table 1. The number of training and test data.

\begin{tabular}{|l|l|l|}
\hline & Training Set & Test Set \\
\hline Normal cell & 40 & 19 \\
\hline Abnormal cell & 40 & 9 \\
\hline Total & 80 & 28 \\
\hline
\end{tabular}

\section{CONCLUSION}

Leukemia is one of the most fatal diseased for the human being. This paper proposed a novel acute leukemia type classification method using CNN as one of the constructing modules for the acute leukemia type clinical decision support system. In the future study, we will consider how to improve our architecture to obtain a better result and try to classify four types of Leukemia.

\section{ACKNOWLEDGEMENTS}

This research was supported by Basic Science Research Program through the National Research F oundation of Korea (NRF) funded by the Ministry of Science and ICT (No. 2016R1D1A3B03931003, No. 2017R1A2B2012456), MSIP (Ministry of Science and ICT), Korea, under the Grand Information Technology Research Center support program (IITP-20172016-0-00318) supervised by the IITP (Institute for Information \& Communications Technology Promotion)", Institute for Information \& communications Technology Promotion(IITP) grant funded by the Korea government(MSIT) (No.2015-0-00225), the Pukyong National University Research Park(PK-URP) for Industry-Academic Convergence R\&D support program 2017, which is funded by the Busan Metropolitan City, Korea, and the Brain Busan 21 Project in 2017.

\section{REFERENCES}

[1] I. Vincent, L. Yang, J.H. Park, P.N. Giao, S.H. Lee, and K.R. Kwon, "Classifying Normal and Abnormal White Blood Cells for Early Detection of Leukemic Disease using Curvelet Transform," in Proceeding of Workshop on Image Processing and Image Understanding 2014, 2014.

[2] L.Putzu and C.D.Ruberto, "White Blood Cells Identification and Counting from Microscopic Blood Image," World Academy of Science, Engineering and Technology, vol. 73, 2013.

[3] R.D.Labati, V.Piuri, and F.Scotti, "ALL-IDB: The Acute Lymphoblastic Leukemia Image Database for Image Processing,” in 18th IEEE International Conference on Image Processing, 2011.

[4] Vasuki Shankar, Mohan Deshpande, N Chaitra, and S Aditi, "Automatic detection of Acute Lymphoblastic Leukemia using Image Processing," in International Conference on Advances in Computer Applications (ICACA), 2016.

[5] Chun-Mei Huo, Hong Zheng, Hong-Yi Su, Zhao-Liang Sun, Yi-Jin Cai, and Yi-Fei Xu, "Tongue Shape Classification Integrating Image Processing and Convolution Neural Network," in 2017 2nd Asia-Pacific Conference on Intelligent Robot Systems, 2017.

[6] Shrutika Mahazan, Snehal S. Golait, Ashwini Meshram, and Nilima Jichlkan, "Review: Detection of types of Acute Leukemia," International Journal of Computer Science and Mobile Computing, vol. 3, no. 3, pp. 104-111, 32014. 


\section{AUTHORS}

Tran Thi Phuong Thanh received the B.S. degree in Information Technology from Water Resources University, Vietnam, in 2015. She is currently studying a master course in IT Convergence and Application Engineering in Pukyong National University, Korea. His research interests include digital signal and image processing, bioinformatics.

Jin-Hyeok Park received the B.S. degree in Computer Software Engineering from Dongeui University, Korea in 2013 and he received an M.S. degree in IT Convergence and Application Engineering in Pukyong National University, Korea in 2015. He is currently studying Ph.D. course in IT Convergence and Application Engineering in Pukyong National University, Korea. His research interests include digital signal and image processing and data compression.

Kwang-Seok Moon He received the B.S., or Studies M.S., and Ph.D degrees in Electronics Engineering in Kyungpook National University, Korea in 1979, 1981, and 1989 respectively. He is 1currently a professor in department of Electronic engineering at Pukyong National University. His research interests include digital image processing, video watermarking, and multimedia communication.

Suk-Hwan Lee received a B.S., an M.S., and a Ph. D. Degrees in Electrical Engineering from Kyungpook National University, Korea in 1999, 2001, and 2004 respectively. He is currently an associate professor in Department of Information Security at Tongmyong University. His research interests include multimedia security, digital image processing, and computer graphics.

Ki-Ryong Kwon received the B.S., M.S., and $\mathrm{Ph}$. D. degrees in electronics engineering from Kyungpook National University in 1986, 1990, and 1994 respectively. He worked at Hyundai Motor Company from 1986-1988 and at the Pusan University of Foreign Language from 1996-2006. He is currently a professor in Dept. of IT Convergence \& Application Engineering at the Pukyong National University. He has researched University of Minnesota in the USA on 2000-2002 with Post-Doc, and Colorado State
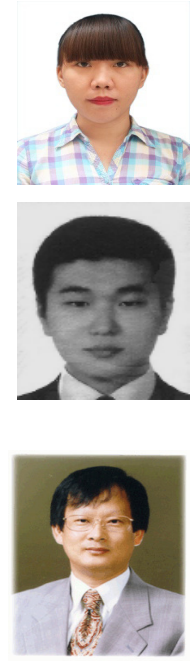
University on 2011-2012 with visiting professor. He was the General President of Korea Multimedia Society on 2015-2016, also was a director of IEEE R10 Changwon section on 2012-2016. His research interests are in the area of digital image processing, multimedia security and watermarking, bioinformatics, weather radar information processing. 